\title{
Regularity of $p$-Harmonic Functions on the Plane
}

Tadeusz Iwaniec and Juan J. Manfredi

\section{Introduction}

Given an open set $\Omega$ in $\mathbb{R}^{n}$, a real-valued function $u$ in the Sobolev class $W_{\text {loc }}^{1, p}(\Omega), 1<p<\infty$, is said to be $p$-harmonic if it is a weak solution of the $p$-harmonic equation

$$
\operatorname{div}\left(|\nabla u|^{p-2} \nabla u\right)=0
$$

or equivalently, for all test functions $\phi \in W^{1, p}(\Omega)$ with compact support in $\Omega$

$$
\int_{\Omega}|\nabla u|^{p-2}\langle\nabla u(x), \nabla \phi(x)\rangle d x=0 .
$$

Note that $p$-harmonic functions are free extremals of the variational integral

$$
I_{G}[u]=\int_{G}|\nabla u(x)|^{p} d x
$$

for each relatively compact open subset $G \subset \Omega$. Two-harmonic functions are real analytic since they are harmonic in the usual sense. When $p \neq 2$ note that equation (1) is non-linear and it degenerates at the zeros of the gradient of $u$. In consequence of this fact $p$-harmonic functions with $p \neq 2$ need not be $C^{\infty}$ smooth. They are however in the Hölder class $C_{\text {loc }}^{1, \alpha}(\Omega)$ with some $\alpha=\alpha(n, p)$, $0<\alpha \leqslant 1$; see Ural'tseva [9], Evans [3] and Lewis [6].

In this note we determine the optimal regularity of $p$-harmonic functions defined on a plane domain for each exponent $p, 1<p<\infty$. To state the results we use the following function spaces. 
The Hölder space $C_{\text {loc }}^{k, \alpha}(\Omega), k=0,1, \ldots, 0<\alpha \leqslant 1$, is the space of complexvalued functions $u \in C^{k}(\Omega)$ whose $k$-th order partial derivatives $D^{\nu} u,|\nu|=k$, are locally Hölder continuous with exponent $\alpha, C_{\mathrm{loc}}^{k, \alpha}(\Omega)$ is a locally convex linear space with topology determined by the seminorms

$$
\|u\|_{C^{k, \alpha(F)}}=\sup _{x \in F}|u(x)|+\sup _{x, y \in F} \sum_{|\nu|=k} \frac{\left|D^{\nu} u(x)-D^{\nu} u(y)\right|}{|x-y|^{\alpha}},
$$

where $F$ is any compact subset of $\Omega$. The completion of $C^{\infty}(\Omega)$ in this topology is a proper subspace of $C_{\text {loc }}^{k, \alpha}(\Omega)$ which we denote by $C_{\mathrm{loc}}^{k+\alpha}(\Omega)$. For $\alpha=1$ the space $C_{\mathrm{loc}}^{k+\alpha}(\Omega)$ coincides with $C^{k+1}(\Omega)$ whereas functions in $C_{\mathrm{loc}}^{k+\alpha}(\Omega)$ with $0<\alpha<1$ are characterized by the condition

$$
\sum_{|\nu|=k}\left|D^{\nu} u(x)-D^{\nu} u(y)\right|=o\left(|x-y|^{\alpha}\right)
$$

uniformly on compact subsets of $\Omega \times \Omega$. Hence, we have the following imbeddings

$$
C_{\mathrm{loc}}^{k+\alpha}(\Omega) \subsetneq C_{\mathrm{loc}}^{k, \alpha}(\Omega) \subsetneq C_{\mathrm{loc}}^{k+\beta}(\Omega) \text {. }
$$

whenever $k=0,1, \ldots$ and $0<\beta<\alpha \leqslant 1$. We denote by $W_{\text {loc }}^{k, s}(\Omega), k=1,2, \ldots$ $1 \leqslant s \leqslant \infty$, the space of functions $u: \Omega \rightarrow \mathbb{C}$ whose distributional derivatives $D^{\nu} u,|\nu| \leqslant k$, belong to $L_{\mathrm{loc}}^{s}(\Omega)$. By Sobolev theorem

$$
W_{\mathrm{loc}}^{k+2, q}(\Omega) \subsetneq W_{\mathrm{loc}}^{k+1, s}(\Omega) \subsetneq C_{\mathrm{loc}}^{k+\alpha}(\Omega)
$$

for $0<\alpha<1$ and $k=0,1,2, \ldots$, where

$$
s=\frac{n}{1-\alpha} \in(n, \infty),
$$

and

$$
q=\frac{n s}{n+s}=\frac{n}{2-\alpha} \in\left(\frac{n}{2}, n\right)
$$

Furthermore

$$
W_{\mathrm{loc}}^{k+1, \infty}(\Omega)=C_{\mathrm{loc}}^{k, 1}(\Omega)
$$

and

$$
W_{\mathrm{loc}}^{k+2, n}(\Omega) \subsetneq W_{\mathrm{loc}}^{k+1, s}(\Omega)
$$

for every $1 \leqslant s<\infty$. Our main result is the following: 
Theorem 1. Let $u \in W_{\mathrm{loc}}^{1, p}(\Omega), 1<p<\infty$, be a $p$-harmonic function defined on a plane domain $\Omega \subset \mathbb{R}^{2}$. Then

$$
u \in C_{\mathrm{loc}}^{k, \alpha}(\Omega) \cap W_{\mathrm{loc}}^{k+2, q}(\Omega),
$$

where the integer $k \geqslant 1$ and the exponent $\alpha \in(0,1]$ are determined by the equation

$$
k+\alpha=\frac{1}{6}\left(7+\frac{1}{p-1}+\sqrt{1+\frac{14}{p-1}+\frac{1}{(p-1)^{2}}}\right) .
$$

The integrability exponent $q$ is any number such that

$$
1 \leqslant q<\frac{2}{2-\alpha} \leqslant 2 .
$$

For $p \neq 2$ the regularity class in (8) is optimal. More precisely, for each $1<p<\infty, p \neq 2$, there is a p-harmonic function $v \in W_{\mathrm{loc}}^{1, p}(\Omega)$ which is not in

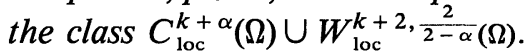

Interest in the regularity problems for $p$-harmonic functions arises from several considerations. One particular compelling connection is with an open problem of Gehring and Reich [4] concerning the degree of summability of the derivatives of a quasiconformal mapping.

Our proof of Theorem 1 substantially exploits and extends the ideas from [2]. A key is the hodograph transformation that converts the $p$-harmonic equation onto a linear first order elliptic system. We solve this system by using Fourier series method. A careful examination of the Fourier expansion formula for the solution of the system leads us to the regularity statement in Theorem 1. This formula provides non-trivial examples of $p$-harmonic functions. Among them there is one showing that our regularity result is the best possible.

\section{Complex Gradient}

We are going to use some properties of plane quasiregular mappings. For this it is convenient to introduce the complex variable

$$
z=x+i y \in \mathbb{C}, \quad(x, y) \in \mathbb{R}^{2},
$$

and the operators of complex differentiation

$$
\frac{\partial}{\partial z}=\frac{1}{2}\left(\frac{\partial}{\partial x}-i \frac{\partial}{\partial y}\right) \text { and } \frac{\partial}{\partial \bar{z}}=\frac{1}{2}\left(\frac{\partial}{\partial x}+i \frac{\partial}{\partial y}\right) .
$$


Let $\Omega$ be an open subset of $\mathbb{C}$. A mapping $f: \Omega \rightarrow \mathbb{C}$ is said to be $K$-quasiregular, $1 \leqslant K<\infty$, if $f \in W_{\mathrm{loc}}^{1,2}(\Omega)$ and

$$
\left|\frac{\partial f(z)}{\partial \bar{z}}\right| \leqslant \frac{K-1}{K+1}\left|\frac{\partial f(z)}{\partial z}\right|
$$

for almost every $z \in \Omega$.

Given a real-valued function $u \in W_{\text {loc }}^{1, p}(\Omega), 1 \leqslant p \leqslant \infty$, the complex gradient of $u$ is defined by

$$
f=\frac{\partial u}{\partial z}=\frac{1}{2}\left(u_{x}-i u_{y}\right)
$$

The complex gradient $f=\partial u / \partial z$ of a $p$-harmonic function $u \in W_{\mathrm{loc}}^{1, p}(\Omega)$, $1<p<\infty$, turns out to be a quasiregular mapping. An essential part of this statement is that $f \in W_{\mathrm{loc}}^{1,2}(\Omega)$. For $p \geqslant 2$ this follows from estimates of the $L^{2}$ modulus of continuity of $\nabla u$, see [2]. The case $1<p<2$ is somewhat delicate and requires an approximation argument, see [8]. The differential inequality (11) follows from (1) without any difficulty. Actually the $p$-harmonic equation can be given the form of one complex equation

$$
\frac{\partial f}{\partial \bar{z}}=\left(\frac{1}{p}-\frac{1}{2}\right)\left[\frac{\bar{f}}{f} \frac{\partial f}{\partial z}+\frac{f}{\bar{f}} \frac{\overline{\partial f}}{\partial z}\right]
$$

Hence, (11) holds with

$$
K=\max \left\{p-1, \frac{1}{p-1}\right\}
$$

Another interesting equation arises for the function $g(z)=|f(z)|^{\sqrt{p-1}-1} f(z)$. It takes the form of Beltrami's equation

$$
\frac{\partial g}{\partial \bar{z}}=\frac{1-\sqrt{p-1}}{1+\sqrt{p-1}} \frac{\bar{g}}{g} \frac{\partial g}{\partial z},
$$

see [2].

In this way the study of $p$-harmonic functions reduces to the study of solutions $f \in W_{\text {loc }}^{1,2}(\Omega)$ of the quasilinear elliptic system (12).

From the theory of quasiregular mappings [1], [5] we find that the complex gradient $f=\partial u / \partial z$ of a $p$-harmonic function $u \in W_{\text {loc }}^{1, p}(\Omega), 1<p<\infty$ is continuous. Moreover, the set $f^{-1}(0)=\{z \in \Omega: \nabla u(z)=0\}$ is discrete provided $u$ is not identically constant. We also know from the general regularity theory concerning elliptic equations that $f$ is $C^{\infty}$-smooth outside the singular set $f^{-1}(0)$. We refer to this result only insofar as it simplifies the arguments used. It is not difficult to dispense with this result since it follows from formula (63). 
For the proof of Theorem 1 it is sufficiently general to examine the regularity of $f$ near one of its zeros, that we may assume is the origin. From now on, $f$ is a $W_{\text {loc }}^{1,2}(\Omega)$-solution of (12), $0 \in \Omega, f(0)=0$.

\section{Hodograph Transformation}

One of the remarkable methods developed for the study of non-linear equations is the hodograph transformation. The idea was originated in an intensive work on non-linear problems in hydrodynamics due mainly to L. Bers and M. A. Lavrentieff. Roughly speaking, given a system of first order differential equations and given a solution $f$ to this system, the hodograph transformation is to write the given system in the hodograph plane in which the independent variable is the inverse of $f$. If the system is quasilinear this simple trick converts it into a linear system with variable coefficients.

Let $f$ be a solution of system (12), $f(0)=0$. Using the factorization theorem for quasiregular mappings [5] we write

$$
f(z)=[\chi(z)]^{n},
$$

where $\chi$ is a quasiconformal homeomorphism defined in a neighborhood of $z=0, \chi(0)=0$, and $n$ is a positive integer.

From (12) we find that

$$
\frac{\partial \chi}{\partial \bar{z}}=\left(\frac{1}{p}-\frac{1}{2}\right)\left[\frac{\chi}{\bar{\chi}} \frac{\overline{\partial \chi}}{\partial z}+\frac{\overline{\chi^{n}}}{\chi^{n}} \frac{\partial \chi}{\partial z}\right] .
$$

Denote by $H=H(\xi)$ the inverse of $\chi=\chi(z)$ in a neighborhood of $z=0$, $z=H(\chi(z)), \xi=\chi(H(\xi)), H(0)=0$. We have

$$
\frac{\partial \chi}{\partial z}=J^{-1} \overline{H_{\xi}}, \quad \frac{\partial \chi}{\partial \bar{z}}=-J^{-1} H_{\bar{\xi}},
$$

where $J(\xi)=\left|H_{\xi}\right|^{2}-\left|H_{\bar{\xi}}\right|^{2}>0$ a.e. since $H$ is quasiconformal in a neighborhood of $\xi=0$. From (16) we see that $H$ satisfies the linear equation

$$
H_{\bar{\xi}}=\left(\frac{1}{2}-\frac{1}{p}\right)\left[\frac{\xi}{\bar{\xi}} H_{\xi}+\frac{\bar{\xi}^{n}}{\xi^{n}} \bar{H}_{\xi}\right] .
$$

Our immediate goal is to solve system (18) in a neighborhood of $\xi=0$. There is no loss of generality assuming that $H$ is defined on the unit disk $\mathbb{B}=$ $\{\xi:|\xi|<1\}$, that $H \in W^{1,2}(\mathbb{B})$ and that $H \in C^{\infty}(\mathbb{B}-\{0\})$. This can be done by rescaling the variable $\xi$, since the function $H(t \xi), t \in \mathbb{R}$, is also a solution to (18). 


\section{Series Expansion for $H(\xi)$}

Theorem 2. Every solution $H \in W^{1,2}(\mathbb{B})$ of equation (18) on the unit disk $\mathbb{B}$ expands into an infinite series of the form

$$
H(\xi)=\sum_{k=n}^{\infty}\left(A_{k} \xi^{k}+\epsilon_{k} \bar{A}_{k} \bar{\xi}^{k}\right)|\xi|^{\lambda_{k}+n-k} \xi^{-n}
$$

where the numbers $\lambda_{k}=\lambda_{k}(n, p)$ and $\epsilon_{k}=\epsilon_{k}(n, p)$ are defined by

$$
\begin{gathered}
2 \lambda_{k}=-n p+\sqrt{4 k^{2}(p-1)+n^{2}(p-2)^{2}}, \\
\epsilon_{k}=\frac{\lambda_{k}+n-k}{\lambda_{k}+n+k}
\end{gathered}
$$

and the complex coefficients $A_{k}, k=n, n+1, \ldots$ satisfy

$$
\sum_{k=n+1}^{\infty} k\left|A_{k}\right|^{2} \leqslant C(n, p) \iint_{\mathbb{B}}\left(\left|H_{\xi}\right|^{2}+\left|H_{\xi}\right|^{2}\right) d \sigma(\xi)<\infty .
$$

The series converges in $W^{1,2}(\mathbb{B})$.

Conversely, given complex numbers $A_{k}, k=n, n+1, \ldots$ satisfying

$$
\sum_{k=n}^{\infty} k\left|A_{k}\right|^{2}<\infty
$$

the series $(19)$ converges in $W^{1,2}(\mathbb{B})$ to a solution of equation (18) for which we have

$$
\iint_{\mathbb{B}}\left(\left|H_{\xi}\right|^{2}+\left|H_{\bar{\xi}}\right|^{2}\right) d \sigma(\xi) \leqslant C(n, p) \sum_{k=n+1}^{\infty} k\left|A_{k}\right|^{2}
$$

Remarks. By elementary considerations the following estimates follow from formulas (20) and (21):

$$
\begin{gathered}
\epsilon_{n}(n, p)=\lambda_{n}(n, p)=0, \\
\left|\epsilon_{k}(n, p)\right|<1, \text { for } k=n+1, n+2, \ldots, \\
\frac{p-1}{n p} \leqslant \lambda_{k}(n, p) \leqslant k \sqrt{p-1}, \text { for } k=n+1, n+2, \ldots
\end{gathered}
$$

If $p=2, \lambda_{k}(n, 2)=k-n$ and $\epsilon_{k}(n, 2)=0, k=n, n+1, \ldots$, thus

$$
H(\xi)=\sum_{k=n}^{\infty} A_{k} \xi^{k-n}
$$

From now on, we assume that $p \neq 2$. 
Proof. It is desirable to use polar coordinates for this problem. Let $0 \leqslant r<1$, $0 \leqslant \theta<2 \pi, \xi=r e^{i \theta}, H(r, \theta)=H\left(r e^{i \theta}\right)$, we have the following transformation formula:

$$
H_{\bar{\xi}}=\frac{1}{2}\left(H_{r}+\frac{i}{r} H_{\theta}\right) e^{i \theta}, \quad H_{\xi}=\frac{1}{2}\left(H_{r}-\frac{i}{r} H_{\theta}\right) e^{-i \theta} .
$$

Since $H$ is $K$-quasiregular,

$$
K=\max \left\{p-1, \frac{1}{p-1}\right\}
$$

then

$$
K^{-1}\left|H_{r}\right|^{2} \leqslant\left|H_{\xi}\right|^{2}-\left|H_{\bar{\xi}}\right|^{2} \leqslant K\left|H_{r}\right|^{2}
$$

and

$$
\left(1+K^{-2}\right)\left|H_{r}\right|^{2} \leqslant 2\left|H_{\xi}\right|^{2}+2\left|H_{\bar{\xi}}\right|^{2} \leqslant\left(1+K^{2}\right)\left|H_{r}\right|^{2} .
$$

Write equation (18) in polar coordinates, conjugate it and eliminate the $\bar{H}_{r}$ term to obtain

$$
2 r H_{r}(r, \theta)=-i p H_{\theta}(r, \theta)+(p-2) i e^{-2 i n \theta} \overline{H_{\theta}(r, \theta)} .
$$

We expand $H(r, \theta)$ into Fourier series with respect to $\theta, 0 \leqslant \theta<2 \pi$.

$$
H(r, \theta)=\sum_{k=-\infty}^{\infty} a_{k}(r) e^{i(k-n) \theta},
$$

where

$$
a_{k}(r)=\frac{1}{2 \pi} \int_{0}^{2 k} H(r, \theta) e^{i(n-k) \theta} d \theta, \quad k \in \mathbb{Z} .
$$

From these formulas, since $H \in C(\mathbb{B}) \cap C^{\infty}(\mathbb{B}-\{0\})$, we see that

$$
a_{k} \in C[0,1) \cap C^{\infty}(0,1), \quad k \in \mathbb{Z} .
$$

Furthermore, since $H \in W^{1,2}(\mathbb{B})$ we are justified to differentiate (29) term by term

$$
\left\{\begin{array}{l}
H_{r}(r, \theta)=\sum_{k=-\infty}^{\infty} a_{k}^{\prime}(r) e^{i(k-n) \theta} \\
H_{\theta}(r, \theta)=i \sum_{k=-\infty}^{\infty}(k-n) a_{k}(r) e^{i(k-n) \theta}
\end{array} .\right.
$$

These series converge in $L^{2}(\mathbb{B})$. 
Formula (30) and equation (28) yield

$$
\begin{aligned}
2 r a_{k}^{\prime}(r)= & \frac{1}{2 \pi} \int_{0}^{2 \pi} 2 r H_{r}(r, \theta) e^{i(n-k) \theta} d \theta \\
= & -i p \frac{1}{2 \pi} \int_{0}^{2 \pi} H_{\theta}(r, \theta) e^{i(n-k) \theta} d \theta+(p-2) i \frac{1}{2 \pi} \int_{0}^{2 \pi} \overline{H_{\theta}(r, \theta)} e^{-i(k+n) \theta} d \theta \\
= & -p(n-k) \frac{1}{2 \pi} \int_{0}^{2 \pi} H(r, \theta) e^{i(n-k) \theta} d \theta \\
& -(p-2)(n+k) \overline{\left(\frac{1}{2 \pi} \int_{0}^{2 \pi} H(r, \theta) e^{i(n+k) \theta} d \theta\right) .}
\end{aligned}
$$

The last equality follows from integration by parts. In view of formulas (30) the right hand integrals are equal to $a_{k}(r)$ and $a_{-k}(r)$, respectively. It leads us to an infinite system of ordinary differential equations for the Fourier coefficients

$$
2 r a_{k}^{\prime}(r)=-p(n-k) a_{k}(r)-(p-2)(n+k) \overline{a_{-k}(r)}
$$

for all $k \in \mathbb{Z}$. Replace $k$ by $-k$ in (33), conjugate it and multiply by $(p-2)$

$$
2(p-2) \overline{r a_{-k}^{\prime}(r)}=-p(p-2)(n+k) \overline{a_{-k}(r)}-(p-2)^{2}(n-k) a_{k}(r) .
$$

We eliminate the term $(p-2)(n+k) \overline{a_{-k}}$ by using (33) again

$$
\begin{aligned}
2(p-2) \overline{r a_{-k}^{\prime}} & =p\left[2 r a_{k}^{\prime}+p(n-k) a_{k}\right]-(p-2)^{2}(n-k) a_{k} \\
& =2 p r a_{k}^{\prime}+4(p-1)(n-k) a_{k} .
\end{aligned}
$$

Finally, we differentiate (33) and, by (34), we obtain

$$
2 r\left(2 r a_{k}^{\prime}\right)^{\prime}=-2 p r(n-k) a_{k}^{\prime}-(n+k)\left[2 p r a_{k}^{\prime}+4(p-1)(n-k) a_{k}\right] .
$$

In this way we arrive at the following uncoupled system of linear (over complex numbers) equations of second order.

$$
r\left(r a_{k}^{\prime}\right)^{\prime}+p n r a_{k}^{\prime}+\left(n^{2}-k^{2}\right)(p-1) a_{k}=0
$$

for $k \in \mathbb{Z}$. Fix $k$, the general solution of (35) must take the form

$$
a_{k}(r)=A^{+} r^{\lambda^{+}}+A^{-} r^{\lambda^{-}},
$$

where $\lambda^{+}$and $\lambda^{-}$are distict roots of the quadratic equation

$$
\lambda^{2}+p n \lambda+\left(n^{2}-k^{2}\right)(p-1)=0,
$$


that is

$$
\begin{aligned}
& 2 \lambda^{+}=-p n+\sqrt{4 k^{2}(p-1)+n^{2}(p-2)^{2}} \\
& 2 \lambda^{-}=-p n-\sqrt{4 k^{2}(p-1)+n^{2}(p-2)^{2}}
\end{aligned}
$$

Since $a_{k} \in C[0,1)$ and $\lambda^{-}<0$, the constant $A^{-}$must vanish. By the same reasoning, the constant $A^{+}$has to be zero whenever $\lambda^{+}$is negative, which happens if $|k|<n$. Hence,

$$
a_{k}(r)=\left\{\begin{array}{ccc}
0 & \text { if } & |k|<n \\
A_{k} r^{\lambda_{k}} & \text { if } & |k| \geqslant n
\end{array},\right.
$$

where

$$
\lambda_{k}=\frac{1}{2}\left(-p n+\sqrt{4 k^{2}(p-1)+n^{2}(p-2)^{2}}\right),
$$

for $k= \pm n, \pm(n+1), \ldots$ Note that $\lambda_{k}=\lambda_{-k}$ and $\lambda_{n}=0$.

We still should verify system (33) because this is not equivalent to (35). Inserting (36) into (33) we find conditions for the coefficients $A_{k}, k= \pm n$, $\pm(n+1), \ldots$,

$$
\left[2 \lambda_{k}+p(n-k)\right] A_{k}=(2-p)(n+k) \overline{A_{-k}} .
$$

For $k= \pm n$, in view of $\lambda_{-n}=\lambda_{n}=0$, we obtain $A_{-n}=0$. There are no restrictions for $A_{n}$. For $|k|>n$ we write (38) as follows

$$
A_{-k}=\epsilon_{k} \overline{A_{k}} \text {, }
$$

where

$$
\epsilon_{k}=\frac{2 \lambda_{k}+p(n-k)}{(2-p)(n+k)}=\frac{\lambda_{k}+n-k}{\lambda_{k}+n+k}
$$

The later identity is computed from formula (37). Observe that $\epsilon_{k} \epsilon_{-k}=1$, thus the change of sign of the index $k$ in (39) leads to the same condition. In conclusion, we may take arbitrary numbers for $A_{n}, A_{n+1}, \ldots$, and determine $A_{-n}, A_{-n-1}, \ldots$ from formula (39).

Returning to the Fourier expansion (29) we write:

$$
H(r, \theta)=\sum_{k=n}^{\infty}\left(A_{k} e^{i k \theta}+\epsilon_{k} A_{k} e^{-i k \theta}\right) r^{\lambda_{k}} e^{-i n \theta},
$$

This is the polar form of (19). A standard computation gives

$$
\int_{0}^{1} r\left(\int_{0}^{2 \pi}\left|H_{r}(r, \theta)\right|^{2} d \theta\right) d r=\pi \sum_{k=n+1}^{\infty} \lambda_{k}\left(1+\epsilon_{k}^{2}\right)\left|A_{k}\right|^{2} .
$$


This, together with estimates (24), (25) and (27), implies (22) with a constant $C(n, p)$ depending only on $n$ and $p$.

Our arguments can be turned back proving the converse statement of Theorem 2.

Corollary 1. Let $H(\xi)$ be a local solution of equation (18) which is quasiconformal in a neighborhood of the origin, $H(0)=0$. Then there is $\rho>0$ and there are constants $0<c<C$ and $C_{m}, m=0,1,2, \ldots$, such that

$$
\begin{gathered}
c|\xi|^{\gamma_{n}} \leqslant|H(\xi)| \leqslant C|\xi|^{\gamma_{n}} \text { for } 0 \leqslant|\xi| \leqslant \rho, \\
c|\xi|^{2 \gamma_{n}-2} \leqslant J(\xi)=\left|H_{\xi}\right|^{2}-\left|H_{\bar{\xi}}\right|^{2} \leqslant C|\xi|^{2 \gamma_{n}-2} \text { for } 0<|\xi| \leqslant \rho,
\end{gathered}
$$

and

$$
\sum_{|\nu|=m}\left|D^{\nu} H(\xi)\right| \leqslant C_{m}|\xi|^{\gamma_{n}-m} \text { for } 0<|\xi| \leqslant \rho
$$

where

$$
\gamma_{n}=\lambda_{n+1}(n, p)=\frac{n}{2}\left\{-p+\sqrt{4\left(1+\frac{1}{n}\right)^{2}(p-1)+(p-2)^{2}}\right\} .
$$

Proof. By rescaling the variable $\xi$ one may assume without loss of generality that $H$ is a quasiconformal solution of (18) on the unit disk, thus it expands into infinite series of the form (19) with $A_{n}=0$. Therefore, for each $0 \leqslant|\xi| \leqslant \rho<1$, we have the following estimates

$$
\begin{aligned}
|H(\xi)| & \leqslant \sum_{k=n+1}^{\infty}\left(1+\left|\epsilon_{k}\right|\right)\left|A_{k}\right||\xi|^{\lambda_{k}} \leqslant 2 \rho^{-\lambda_{n+1}}|\xi|^{\lambda_{n+1}} \sum_{k=n+1}^{\infty}\left|A_{k}\right| \rho^{\lambda_{k}} \\
& \leqslant 2 \rho^{-\lambda_{n+1}}|\xi|^{\lambda_{n+1}}\left(\sum_{k=n+1}^{\infty} k\left|A_{k}\right|^{2}\right)^{1 / 2}\left(\sum_{k=n+1}^{\infty} k^{-1} \rho^{2 \lambda_{k}}\right)^{1 / 2} \leqslant C|\xi|^{\gamma_{n}} .
\end{aligned}
$$

The convergence of the last two series is verified by (22) and (25). These arguments also show that the series (19) converges uniformly, on $\{\xi:|\xi| \leqslant \rho\}$. Applying argument principle, we find that $A_{n+1} \neq 0$. Hence for $0 \leqslant|\xi| \leqslant \rho<1$, we obtain

$$
\begin{aligned}
|H(\xi)| & \geqslant\left(1-\left|\epsilon_{n+1}\right|\right)\left|A_{n+1}\right||\xi|^{\lambda_{n+1}}-\sum_{k=n+2}^{\infty}\left(1+\left|\epsilon_{k}\right|\right)\left|A_{k}\right||\xi|^{\lambda_{k}} \\
& \geqslant|\xi|^{\lambda_{n+1}}\left[\left(1-\left|\epsilon_{n+1}\right|\right)\left|A_{n+1}\right|-2 \rho^{\lambda_{n+2}-\lambda_{n+1}} \sum_{k=n+2}^{\infty}\left|A_{k}\right| \rho^{\lambda_{k}-\lambda_{n+2}}\right] .
\end{aligned}
$$

For suffiently small $\rho$, the expression in rectangular parantheses is positive. Hence (41) follows. To prove (43) we note that $\lim \lambda_{k}=\infty$. Therefore, we can 
perform any finite number of term-by-term differentiations in formula (19) for $0<|\xi|<1$. This gives the required estimate

$$
\begin{aligned}
\sum_{|\nu|=m}\left|D^{\nu} H(\xi)\right| \leqslant & C(n, p, m) \sum_{k=n+1}^{\infty} \lambda_{k}^{m}\left|A_{k}\right||\xi|^{\lambda_{k}-m} \\
\leqslant & C(n, p, m)|\xi|^{\lambda_{n+1}-m} \sum_{k=n+1}^{\infty} k^{m}\left|A_{k}\right| \rho^{\lambda_{k}-\lambda_{n+1}} \\
\leqslant & C(n, p, m)|\xi|^{\lambda_{n+1}-m}\left[\sum_{k=n+1}^{\infty} k\left|A_{k}\right|^{2}\right]^{1 / 2} \\
& \times\left[\sum_{k=n+1}^{\infty} k^{2 m-1} \rho^{2 \lambda_{k}-2 \lambda_{n+1}}\right]^{1 / 2}
\end{aligned}
$$

For $m=1$, this implies the upper bound of $J(\xi)$ as stated in (42). To prove the lower bound for $J(\xi)$, we use expansion (40) from which it follows that

$$
H_{r}(r, \theta)=\sum_{k=n+1}^{\infty} \lambda_{k}\left(A_{k} e^{i k \theta}+\epsilon_{k} \overline{A_{k}} e^{-i k \theta}\right) e^{-i n \theta} r^{\lambda_{k}-1} .
$$

Hence, for $0<r=|\xi| \leqslant \rho<1$ we find

$$
\begin{aligned}
\left|H_{r}\right| & \geqslant \lambda_{n+1}\left(1-\left|\epsilon_{n+1}\right|\right)\left|A_{n+1}\right| r^{\lambda_{n+1}-1}-\sum_{k=n+2}^{\infty} \lambda_{k}\left(1+\left|\epsilon_{k}\right|\right)\left|A_{k}\right| \rho^{\lambda_{k}-1} \\
& \geqslant r^{\lambda_{n+1}-1}\left(\lambda_{n+1}\left(1-\left|\epsilon_{n+1}\right|\right)\left|A_{n+1}\right|-2 \rho^{\lambda_{n+2}-\lambda_{n+1}} \sum_{K=n+2}^{\infty} \lambda_{k}\left|A_{k}\right| \rho^{\lambda_{k}-\lambda_{n+2}}\right) \\
& =c(n, p, \rho) r^{\gamma_{n}-1},
\end{aligned}
$$

where, in view of (22) and (25), the constant $c(n, p, \rho)$ is positive as $\rho$ gets small. This together with inequality (26) implies

$$
J(\xi)=\left|H_{\xi}\right|^{2}-\left|H_{\bar{\xi}}\right|^{2} \geqslant c|\xi|^{2 \gamma_{n}-2} \text { for } 0<|\xi| \leqslant \rho .
$$

\section{Estimates of the Derivatives of $f(z)$}

We return to the function $f(z)=[\chi(z)]^{n}$, where $\chi=\chi(z)$ is the inverse to $H(\xi)$, that is

$$
\chi(H(\xi))=\xi
$$

for $|\xi| \leqslant \rho$. We shall express the partial derivatives $D^{\nu} \chi(z),|\nu|=0,1, \ldots$, at $z=H(\xi)$ in terms of $D^{\mu} H(\xi),|\mu|=0,1, \ldots$ The first order derivatives of $\chi$ 
are easy to derive from (45):

$$
\left\{\begin{array}{l}
\chi_{z}(z)=J^{-1}(\xi) \overline{H_{\xi}} \\
\chi_{\bar{z}}(z)=-J^{-1}(\xi) H_{\bar{\xi}}
\end{array} .\right.
$$

To describe the formulas for higher derivatives of $\chi(z)$ we need the following convention. Given a positive integer $s$ let $H^{s}$ denote one of the partials

$$
\frac{\partial^{s} H}{\partial \xi^{i} \partial \xi^{j}} \text { or } \frac{\overline{\partial^{s} H}}{\partial \xi^{i} \overline{\partial \xi^{j}}},
$$

where $i$ and $j$ run over non-negative integers such that $i+j=s$. For example, the symbol $H^{1}$ stands for one of the first order derivatives $H_{\xi}, H_{\bar{\xi}}, \overline{H_{\xi}}$ or $\overline{H_{\bar{\xi}}}$ and we do not specify which one of them. Any expression of the form $H^{s_{1}} H^{s_{2}} \cdots H^{s_{k}}$, where $s_{1}+s_{2}+\cdots+s_{k}=s$ will be called a monomial of type $(s, k)$.

The set of all linear combinations of monomials of type $(s, k)$ is denoted by $\mathbb{P}(s, k), s=1,2, \ldots, k=1,2, \ldots$ For instance, the Jacobian determinant $J(\xi)=H_{\xi} \bar{H}_{\xi}-H_{\bar{\xi}} \bar{H}_{\bar{\xi}}$ is a member of $\mathbb{P}(2,2)$. Let us remark that

(i) If $P \in \mathbb{P}(s, k)$, then $P_{\xi}, P_{\bar{\xi}} \in \mathbb{P}(s+1, k)$,

(ii) $J_{\xi}=H_{\xi \xi} \overline{H_{\xi}}+\overline{H_{\bar{\xi} \xi}} H_{\xi}-H_{\bar{\xi} \bar{\xi}} \overline{H_{\bar{\xi}}}-\overline{H_{\bar{\xi} \xi}} H_{\bar{\xi}} \in \mathbb{P}(3,2)$,

(iii) if $P \in \mathbb{P}(s, k)$ and $Q \in \mathbb{P}(t, l)$, then $P Q \in \mathbb{P}(s+t, k+l)$.

Now we generalize formula (46).

Lemma 1. Let $|\nu|=m, m=1,2, \ldots$, then there exists

$$
P=P_{\nu} \in \mathbb{P}(4 m-3,3 m-2)
$$

such that

$$
D^{\nu} \chi(z)=J(\xi)^{1-2 m} P_{\nu}(\xi),
$$

where $z=H(\xi)$.

Proof. We perform induction with respect to $m$. For $m=1$ formula (47) follows from (46). Suppose that (47) holds for some integer $m \geqslant 1$. Applying chain rule, we obtain

$$
\left\{\begin{array}{l}
\left(D^{\nu} \chi\right)_{z} H_{\xi}+\left(D^{\nu} \chi\right)_{\bar{z}} \overline{H_{\bar{\xi}}}=J^{-2 m}\left[J P_{\xi}+(1-2 m) P J_{\xi}\right] \\
\left(D^{\nu} \chi\right)_{z} H_{\bar{\xi}}+\left(D^{\nu} \chi\right)_{\bar{z}} \overline{H_{\xi}}=J^{-2 m}\left[J P_{\bar{\xi}}+(1-2 m) P J_{\bar{\xi}}\right]
\end{array}\right.
$$

This system can be solved for partials $\left(D^{\nu} \chi\right)_{z}$ and $\left(D^{\nu} \chi\right)_{\bar{z}}$ :

$$
\begin{aligned}
& \left(D^{\nu} \chi\right)_{z}=J^{-1-2 m}\left[J P_{\xi} \overline{H_{\xi}}-J P_{\bar{\xi}} \overline{H_{\bar{\xi}}}+(1-2 m) P J_{\xi} \overline{H_{\xi}}-(1-2 m) P J_{\bar{\xi}} \overline{H_{\bar{\xi}}}\right], \\
& \left(D^{\nu} \chi\right)_{\bar{z}}=J^{-1-2 m}\left[J P_{\bar{\xi}} H_{\xi}-J P_{\xi} H_{\bar{\xi}}+(1-2 m) P J_{\bar{\xi}} H_{\xi}-(1-2 m) P J_{\xi} H_{\bar{\xi}}\right] .
\end{aligned}
$$


What remains to be established is that the expressions in the brackets [...] are members of $\mathbb{P}(4(m+1)-3,3(m+1)-2)=\mathbb{P}(4 m-1,3 m+1)$. One can verify this fact by using the induction hypothesis and properties (i-iii). Thus formula (47) holds for $m+1$.

Combining Lemma 1 together with Corollary 1 we obtain estimates for the derivatives of the complex gradient $f=f(z)$.

Corollary 2. Suppose $f(z)$ is a local solution to the system (12) which has the form (15), $f(0)=0$. Then there is $\delta>0$ and there are constants $B_{m}$, $m=0,1,2, \ldots$, such that

$$
\sum_{|\nu|=m}\left|D^{\nu} f(z)\right| \leqslant B_{m}|z|^{n / \gamma_{n}-m},
$$

for $0<|z|<\delta$, where $\gamma_{n}$ is defined by (44).

Proof. First we examine the quasiconformal map $\chi=\chi(z)$. Fix $|\nu|=l$, $l=1,2, \ldots, m$. By Lemma 1 ,

$$
D^{\nu} \chi(z)=J(\xi)^{1-2 l} P_{\nu}(\xi),
$$

for $0<|\xi|<\rho$, where $P_{\nu} \in \mathbb{P}(4 l-3,3 l-2)$. In view of inequality (43) any monomial $Q=H^{s_{1}} H^{s_{2}} \cdots H^{s_{k}}, s_{1}+\cdots+s_{k}=s$, of type $(s, k)$ admits an estimate

$$
|Q(\xi)| \leqslant M|\xi|^{\gamma_{n}-s_{1}}|\xi|^{\gamma_{n}-s_{2}} \cdots|\xi|^{\gamma_{n}-s_{k}}=M|\xi|^{k \gamma_{n}-s},
$$

for $0<|\xi|<\rho$. Here and below the letter $M$ stands for a constant independent of $\xi$, not necessarily the same in each appearance. Since $P_{\nu}(\xi)$ is a linear combination of monomials of type $(4 l-3,3 l-2)$, we then find that

$$
\left|P_{\nu}(\xi)\right| \leqslant M|\xi|^{(3 l-2) \gamma_{n}-4 l+3},
$$

for $0<|\xi|<\rho$. On the other hand, using (42), we see that

$$
J(\xi)^{1-2 l} \leqslant M|\xi|^{(1-2 l)\left(2 \gamma_{n}-2\right)} .
$$

The above estimates together with formula (49) imply

$$
\left|D^{\nu} \chi(z)\right| \leqslant M|\xi|^{1-l \gamma_{n}},
$$

where $|\nu|=l, l=0,1,2, \ldots, m$. This also holds true for $l=0$ since $\chi(z)=\xi$.

According to (41), $c|\xi|^{\gamma_{n}} \leqslant|z| \leqslant C|\xi|^{\gamma_{n}}$, so for each $|\nu|=l, l=0,1, \ldots, m$ we have

$$
\left|D^{\nu} \chi(z)\right| \leqslant M|z|^{1 / \gamma_{n}-l}
$$

for $0<|z|<\delta$, with sufficiently small $\delta>0$. 
Finally, applying Leibniz formula for the derivatives of $f(z)=[\chi(z)]^{n}$ we come to the required estimate $\left|D^{\nu} f(z)\right| \leqslant M|z|^{n / \gamma_{n}-m}$ for each $|\nu|=m$.

\section{Proof of the Regularity Statement}

Let $u \in W_{\mathrm{loc}}^{1, p}(\Omega), 1<p<\infty, p \neq 2$, be a $p$-harmonic function. As mentioned before, it suffices to establish the regularity of $u$ near one of its singular points, that we may assume is the origin. Denote by $f=f(z)$ the complex gradient of $u, f(0)=0$. Corollary 2 applies to $f$ for some positive integer $n$. By formula (44) we see that the smallest value of the numbers $n / \gamma_{n}, n=1,2, \ldots$, occurs for $n=1$. Let this minimum value be denoted by $d=d(p), 1<p<\infty$.

$$
d=\frac{1}{6}\left(1+\frac{1}{p-1}+\sqrt{1+\frac{14}{p-1}+\frac{1}{(p-2)^{2}}}\right) .
$$

Hence, we obtain estimates which are independent of $n$

$$
\sum_{|\nu|=m}\left|D^{\nu} f(z)\right| \leqslant B_{m}|z|^{d-m},
$$

or equivalently

$$
\sum_{|\nu|=m+1}\left|D^{\nu} u(z)\right| \leqslant B_{m}|z|^{d-m},
$$

for $0<|z|<\delta$ and $m=0,1,2, \ldots$

Let the integer $k$ and the exponent $\alpha \in(0,1]$ be determined by (9), so $d=k-1+\alpha$. Take $m$ in (52) equal to $k+1$

$$
\sum_{|\nu|=k+2}\left|D^{\nu} u(z)\right| \leqslant B_{k+1}|z|^{\alpha-2} \text { for } 0<|z|<\delta .
$$

The function $|z|^{\alpha-2}$ is integrable over the disk $0 \leqslant|z|<\delta$ with every exponent $q$ such that $1 \leqslant q<2 /(2-\alpha)$. Hence $u \in W_{\mathrm{loc}}^{k+2, q}(\Omega)$.

These two estimates imply Hölder's condition of exponent $\alpha$ for functions $D^{\nu} u(z),|\nu|=k$. This fact follows from an elementary lemma.

Lemma 2. Let $U$ be a convex open subset in $\mathbb{R}^{n}$ containing the origin and let $F$ be a function of class $C(U) \cap C^{1}(U-\{0\})$ such that

(i) $|F(x)| \leqslant M|x|^{\alpha}$ for $x \in U$,

(ii) $|\nabla F(x)| \leqslant M|x|^{\alpha-1}$ for $x \in U-\{0\}$,

where $\alpha \in(0,1]$. Then

$$
|F(x)-F(y)| \leqslant 5 M|x-y|^{\alpha}, \text { for all } x, y \in U .
$$


Proof. Two cases are possible.

Case 1. Suppose that $|y| \leqslant 2|x-y|$, then

$$
\begin{aligned}
|F(x)-F(y)| & \leqslant|F(x)|+|F(y)| \leqslant 2^{1-\alpha}\left(|F(x)|^{1 / \alpha}+|F(y)|^{1 / \alpha}\right)^{\alpha} \\
& \leqslant 2^{1-\alpha} M(|x|+|y|)^{\alpha} \leqslant 2^{1-\alpha} M(|x-y|+2|y|)^{\alpha} \\
& \leqslant 2^{1-\alpha} 5^{\alpha} M|x-y|^{\alpha} \leqslant 5 M|x-y|^{\alpha} .
\end{aligned}
$$

Case 2. Suppose that $|y| \geqslant 2|x-y|>0$, then for each $0 \leqslant t \leqslant 1$ we have

$$
|t x+(1-t) y| \geqslant|y|-t|x-y| \geqslant|x-y| \text {. }
$$

Thus

$$
|t x+(1-t) y|^{\alpha-1} \leqslant|x-y|^{\alpha-1}
$$

Using this estimate, we conclude

$$
\begin{aligned}
|F(x)-F(y)| & =\left|\int_{0}^{1} \frac{d}{d t} F(t x+y-t y) d t\right|=\left|\int_{0}^{1}\langle x-y, \nabla F(t x+y-t y)\rangle d t\right| \\
& \leqslant M \int_{0}^{1}|x-y||t x+(1-t) y|^{\alpha-1} d t \leqslant M|x-y|^{\alpha} .
\end{aligned}
$$

\section{The Extremal Function}

Take $n=1$ in (15) and $A_{1}=0, A_{2}=1, A_{3}=A_{4}=\cdots=0$ in the series (19). In this case

$$
\lambda_{2}=\gamma_{1}=\frac{1}{d} \quad \text { and } \quad \epsilon_{2}=\frac{\lambda_{2}-1}{\lambda_{2}+3}=\frac{1-d}{1+3 d}
$$

We then have

$$
H(\xi)=\left(\frac{\xi}{|\xi|}+\epsilon \frac{|\xi|^{3}}{\xi^{3}}\right)|\xi|^{1 / d}, \quad \epsilon=\frac{1-d}{1+3 d}
$$

Note that for $p=2$, in view of formula (44), we have $d=1, \epsilon=0$, and $H(\xi)=\xi$. It is of crucial importance that $\epsilon \neq 0$ for $p \neq 2$. In this case $H$ is a quasiconformal homeomorphism on the whole plane.

Let $f=f(z)$ denote the inverse of $H=H(\xi)$

$$
f(H(\xi))=\xi, \quad z=H(\xi), \quad \xi=f(z) .
$$


This defines a $p$-harmonic function $v=W_{\text {loc }}^{1, p}(\mathbb{C})$ of complex gradient equal to $f, \partial v / \partial z=f(z)$. We complete the proof of Theorem 1 by showing that

$$
f \notin C_{\mathrm{loc}}^{k-1+\alpha}(\mathbb{C}) \cup W_{\mathrm{loc}}^{k+1, q}(\mathbb{C}), \quad q=\frac{2}{2-\alpha} .
$$

For this effect let us remark that $f$ is a homogeneous function of degree $d$,

$$
f(t z)=t^{d} f(z), \quad z \in \mathbb{C} \text { and } t \geqslant 0 .
$$

Indeed, by (54), $t H(\xi)=H\left(t^{d} \xi\right)$, and by (55)

$$
f(t z)=f(t H(\xi))=f\left(H\left(t^{d} \xi\right)\right)=t^{d} \xi=t^{d} f(z) .
$$

Hence, partial derivatives $D^{\nu} f$ are homogeneous functions of degree $d-|\nu|$. In particular, since $d=k-1+\alpha$, we have

$$
\left(D^{\nu} f\right)(t z)=t^{\alpha} D^{\nu} f(z)
$$

for $z \neq 0, t>0$ and $|\nu|=k-1$, and

$$
D^{\nu} f(z)=t^{2-\alpha}\left(D^{\nu} f\right)(t z),
$$

for $z \neq 0, t>0$ and $|\nu|=k+1$. Now, suppose to the contrary that (56) fails. We have two cases:

Case 1. If $f \in W_{\mathrm{loc}}^{k+1, q}(\mathbb{C})$, then integration of (59) gives

$$
\iint_{|z|<R}\left|D^{\nu} f(z)\right|^{q} d \partial(z)=\iint_{|z|<t R}\left|D^{\nu} f(z)\right|^{q} d \partial(z)
$$

for all positive $t$ and $R$. Hence $D^{\nu} f(z)=0$ for each $|\nu|=k+1$.

Case 2. If $f \in C_{\mathrm{loc}}^{k-1+\alpha}(\mathbb{C})$, then, by (58) and by the definition of the class $C_{\text {loc }}^{k-1+\alpha}(\mathbb{C})$, we find that

$$
D^{\nu} f(z)=\lim _{t \rightarrow 0} t^{-\alpha} D^{\nu} f(t z)=\left\{\begin{array}{crr}
0 & \text { if } & 0<\alpha<1 \\
z\left(D^{\nu} f\right)_{z}(0)+\bar{z}\left(D^{\nu} f\right)_{\bar{z}}(0) & \text { if } & \alpha=1
\end{array}\right.
$$

for each $|\nu|=k-1$.

In each case, we conclude that $f$ must be a homogeneous polynomial. The number $d$ in (57) is therefore a positive integer and

$$
f(z)=\sum_{m+n=d} a_{m n} z^{m} \bar{z}^{n},
$$


or, equivalently,

$$
\xi=\sum_{m+n=d} A_{m n} H(\xi)^{m} \overline{H(\xi)^{n}} .
$$

We use this formula only for $|\xi|=1$, in which case $H(\xi)=\xi+\epsilon \xi^{-3}$ and $\bar{H}(\xi)=\xi^{-1}+\epsilon \xi^{3}$. Thus,

$$
\xi=\sum_{m+n=d} A_{m n}\left(\xi+\epsilon \xi^{-3}\right)^{m}\left(\xi^{-1}+\epsilon \xi^{3}\right)^{n}
$$

This identity admits analytic continuation to all $\xi \neq 0$. In particular, we may evaluate (60) at each of the four possible values of the root $\sqrt[4]{-\epsilon}$ getting $(\sqrt[4]{-\epsilon})^{d+1}=A_{0 d}\left(1-\epsilon^{2}\right)^{d}$. This yields $d+1 \equiv 0(\bmod 4)$. On the other hand (60) evaluated at each of the four values of $\sqrt[4]{-1 / \epsilon}$ gives

$$
\left(\sqrt[4]{\frac{-1}{\epsilon}}\right)^{3 d+1}=A_{d 0}\left(\epsilon-\frac{1}{\epsilon}\right)^{d}
$$

which yields $3 d+1=0(\bmod 4)$. Contradiction arises since $(d+1)+(3 d+1)$ $\equiv 2(\bmod 4)$.

The proof of Theorem 1 is complete.

\section{Notes and Remarks}

Let us perform hodograph transformation of system (14) for the function $g(z)=|f(z)|^{\sqrt{p-1}-1} f(z), f(0)=0$. The local solutions of (14) take the form

$$
g(z)=[y(z)]^{n},
$$

where $n$ is a positive integer and $y$ is a quasiconformal mapping in a neighborhood of the origin. The corresponding system for $y(z)$ can be written as follows:

$$
\frac{\partial y}{\partial \bar{z}}=\frac{1-\sqrt{p-1}}{1+\sqrt{p-1}} \frac{\bar{y}^{n}}{y^{n}} \frac{\partial y}{\partial z} .
$$

Denote by $F=F(\xi)$ the inverse of $y=y(z)$. Then (61) converts onto a linear system

$$
F_{\bar{\xi}}(\xi)=\frac{\sqrt{p-1}-1}{\sqrt{p-1}+1} \frac{\bar{\xi}^{n}}{\xi^{n}} \overline{F_{\xi}(\xi)} .
$$

An advantage in studying this system is that after an elementary substitution

$$
W(\xi)=\left(\xi^{n} F-\frac{\sqrt{p-1}-1}{\sqrt{p-1}+1} \bar{\xi}^{n} \bar{F}\right)|\xi|^{-1+p / 2 \sqrt{p-1}},
$$


one obtains a very simple system of Cauchy-Riemann type

$$
W_{\bar{\xi}}(\xi)=\frac{p-2}{4 \sqrt{p-1}} \frac{1}{\xi} \overline{W(\xi)} .
$$

In particular, successive differentiation of (63) leads to the $C^{\infty}$-regularity result outside $\xi=0$. To finish, we remark that the complex gradient $f=u_{z}$ of a $p$-harmonic function $u \in W_{\mathrm{loc}}^{1, p}(\Omega)$ is a $K$-quasiregular mapping with

$$
K=\max \left\{p-1, \frac{1}{p-1}\right\}
$$

It is known that $K$-quasiregular mappings are locally Hölder continuous with exponent $K^{-1}$, [1], [5]. In our case, it would give $f \in C_{\mathrm{loc}}^{\beta}(\Omega)$ with

$$
\beta=\min \left\{p-1, \frac{1}{p-1}\right\}
$$

However, for the complex gradient $f=u_{z}$ we have a better result since $f \in$ $C_{\mathrm{loc}}^{k-1, \alpha}(\Omega)$ and by (9) and (50)

$$
\begin{aligned}
& k-1+\alpha=d(p)=\frac{1}{6}\left(1+\frac{1}{p-1}+\sqrt{1+\frac{14}{p-1}+\frac{1}{(p-1)^{2}}}\right) \\
&>\min \left\{p-1, \frac{1}{p-1}\right\} \text { for } 1<p<\infty, \quad p \neq 2 .
\end{aligned}
$$

A well known conjecture in quasiconformal analysis [4] asserts that plane $K$-quasiregular mappings belong to $W_{\mathrm{loc}}^{1, s}(\Omega)$ for any $2<s<2+2 /(K-1)$. For the complex gradient $f$ of a $p$-harmonic function, $p \neq 2$, we would obtain

$$
f \in W_{\mathrm{loc}}^{1, s}(\Omega),
$$

for any

$$
2<s<\left\{\begin{array}{lll}
\frac{2 p-2}{p-2} & \text { if } & 2<p<\infty \\
\frac{2}{2-p} & \text { if } & 1<p<2
\end{array} .\right.
$$

However, for this special class of quasiregular mappings, Theorem 1 states a better result.

NotE ADDED IN PROOF. We have received a preprint of G. Aronsson «Representation of a $p$-harmonic function in the plane», in which he obtains, 
via stream functions, representation of a $p$-harmonic function $u$ near a singular point. As a corollary of this representation he also obtains a Hölder regularity result. This result, according to Theorem 1 , is sharp if $p>2$.

\section{References}

[1] Ahlfors, L. Lectures on Quasiconformal Mappings, Von Nostrand Company, 1966.

[2] Bojarski, B. and Iwaniec, T. p-harmonic equation and quasiregular mappings, Preprint, Universität Bonn.

[3] Evans, L. A new proof of Local $C^{1, \alpha}$ regularity for solutions of certain degenerated elliptic P.D.E., J. Diff. Equations, 45(1982), 356-373.

[4] Gehring, F. and Reich, E. Area distorsion under quasiconformal mappings, Ann. Acad. Sci. Fenn. Ser. A, 388(1966), 1-15.

[5] Lehto, O. and Virtanen, K. Quasiconformal mappings in the plane, SpringerVerlag, 1973.

[6] Lewis, L. Smoothness of certain degenerate elliptic equations, Proc. Amer. Math. Soc. 80(1980), 259-265.

[7] Lewis, J. Regularity of the derivatives of solutions to certain elliptic equations, Indiana Univ. Math. J. 32(1983), 849-858.

[8] Manfredi, J. $p$-Harmonic Functions in the Plane, Proc. Amer. Math. Soc. 103(1988), 473-479.

[9] Ural'tseva, N. Degenerate quasilinear elliptic systems, Zap. Naucn. Sem. 7(1968), 184-222. Otdel. Math. Inst. Steklov, Leningrad (in Russian).

Recibido: 12 de agosto de 1988.

Tadeusz Iwaniec*

Department of Mathematics

Syracuse University

200 Carnegie Bldg.

Syracuse, NY 13210

U.S.A.
Juan J. Manfredi**

Department of Mathematics

Purdue University

West Lafayette, IN 47907

U.S.A.

* Supported in part by NSF Grant DMS-8807924.

** Supported in part by NSF Grant DMS-8703286. 